\title{
Inherited predisposition to pancreatic cancer
}

Pancreatic cancer is the fifth leading cause of death from cancer in men and women in Europe and North America. The prognosis has not changed significantly despite improvements in diagnosis and perioperative management. Traditional chemotherapy and radiotherapy regimens have been rather ineffective in providing control of the disease. ${ }^{1}$

The only consistent risk factor identified so far is smoking and possibly accounts for a quarter of all cases of pancreatic cancer. ${ }^{2}$ Diabetes mellitus is another factor associated with an increased risk for the development of pancreatic cancer although it is still unclear if diabetes is a cause or an effect of malignant disease in the organ. ${ }^{3}$ Surgical resection offers the best chance of prolongation in survival. Unfortunately, in more than $80 \%$ of cases, at the time of diagnosis the cancer is either locally advanced or disseminated thus excluding curative resection. Hope for making an appreciable impact on survival has been focused on two areas: early diagnosis of pancreatic precancer and introduction of new therapeutic modalities based on the molecular and genetic abnormalities identified in the disease. ${ }^{4}$

Studies have shown an increased risk of pancreatic cancer among relatives of patients with pancreatic cancer $(7.8 \%$ compared with $0.6 \%$ in controls), with no apparent differences in the environmental risk exposures observed in the two groups. ${ }^{5}$ Similar observations were made by Fernandez et al in a study in which 362 histologically confirmed pancreatic cancer cases were compared with 1408 patients admitted to hospital for acute, benign, non-digestive tract disorders. A significant association was noted between a family history of pancreatic cancer and the risk of developing pancreatic cancer (odds ratio 3.0, 95\% confidence intervals 1.4-6.6). Even after adjustment for factors such as tobacco consumption, alcohol intake, pancreatitis, and diabetes, the risk of pancreatic cancer remained almost the same in patients with a positive family history (odds ratio $2.8,95 \%$ confidence intervals $1.3-6.3) .{ }^{6}$ The same study suggested the existence of a genetic component in 3\% of newly diagnosed pancreatic cancers. Two recent studies, one population based and the other derived from the National Familial Pancreas Tumor Registry, revealed that cancer risk increases in first and second degree relatives when there are two or more affected members in a family (odds ratio approximating 3.5). ${ }^{37}$

Some cases of pancreatic cancer exhibit a definite familial clustering and it is estimated that $5-10 \%$ of cases are due to hereditary factors. ${ }^{8-15}$ At the University of Washington the following selection criteria have been used to identify individuals with an increased risk of developing pancreatic cancer in a pancreatic cancer surveillance programme:

- an individual with two or more first degree relatives with pancreatic adenocarcinoma,

- an individual with one first degree relative who developed pancreatic adenocarcinoma at a young age (younger than 50 years),
- an individual with two or more second degree relatives with pancreatic adenocarcinoma, one of whom developed the cancer at an early age. ${ }^{16}{ }^{17}$

The increased risk of pancreatic cancer in pancreatic cancer prone families is associated either as part of a known syndrome, where predisposition to pancreatic cancer is one part of the cancer spectrum that these families develop, or alternatively it can occur as the only type of cancer that is inherited.

\section{$B R C A 2$ gene germline mutation and pancreatic cancer}

$B R C A 2$ germline mutation carriers have an increased risk of male or female breast cancer and pancreatic cancer, the lifetime risk of breast cancer being approximately $25 \% .{ }^{18}$ In a study of 220 Jewish families affected by female breast cancer, the frequency of $B R C A 2$ germline mutations was $4.1 \%{ }^{19}$ Of these 220 Jewish families, 19 had a history of pancreatic cancer although histological confirmation was not available and the mutation status of these patients was not tested. Germline mutations were present in $14(73.7 \%)$ of these families (11 in BRCA1 and three in BRCA2) while in families without pancreatic cancer the incidence of mutations in either of the two genes was $42.8 \%$. In the same study, a family history of pancreatic cancer was found to be a strong predictor of the presence of the $B R C A 2$ mutation (odds ratio $6.1 ; \mathrm{p}=0.03$ ). The lack of histological confirmation and unknown mutation status of patients who developed pancreatic cancer in these families necessitates cautious interpretation of the results.

Thorlacius et al investigated the frequency of a $B R C A 2$ germline mutation in 21 Icelandic families with male and female breast cancer. ${ }^{20}$ In 16 of these families with the $B R C A 2$ (999del5) germline mutation, there were 11 cases of pancreatic cancer. Conversely, there were no cases of pancreatic cancer in the mutation negative group. Phelan et al studied 49 site specific breast cancer families and found $B R C A 2$ mutations in eight families (four of which had a positive family history for male breast cancer). In this study, the presence of pancreatic cancer was reported more often in the mutation positive families (four of eight) than in the mutation negative families (five of 41), again indicating an association between $B R C A 2$ mutations and pancreatic cancer ( $R R$ 7.2; $\mathrm{p}=0.03$ ). Interestingly, age of onset for the four pancreatic cancer cases in the families carrying the BRCA2 mutation was significantly lower than the mutation negative families. None of these four cases

Abbreviations used in this paper: FAMMM, familial atypical multiple mole melanoma syndrome; HNPCC, hereditary non-polyposis colorectal carcinoma; PJS, Peutz-Jeghers syndrome; $\mathrm{LOH}$, loss of heterozygosity; PanIN, pancreatic intraepithelial neoplasia; EUS, endoscopic ultrasound; MRCP, magnetic resonance cholangiopancreatography 
had DNA tested for mutation in the BRCA2 gene although for two, haplotype analysis indicated that they were obligate mutation carriers. ${ }^{21}$

It is apparent that $B R C A 2$ germline mutations are associated with low penetrance of both breast and pancreatic cancer, such that many kindred who carry the mutation do not develop cancer. Goggins et al detected germline $B R C A 2$ mutations in $7.3 \%$ (3/41, two of which were 6174 delT) of apparently sporadic pancreatic cancers and none of these patients had a family history suggestive of predisposition to cancer. ${ }^{22}$ Likewise, Ozcelik et al investigated the contribution of germline BRCA2 mutation in 42 cases of pancreatic cancer without a family history and identified two $(4.9 \%)$ germline mutations. The same group investigated 39 Jewish patients with pancreatic cancer for germline BRCA2 6174delT mutation and found four mutation positive cases $(10 \%)$. This frequency is significantly higher than that of the germline BRCA2 6174delT in the general Ashkenazi population (1.36\%). They estimated the risk of acquiring pancreatic cancer by the age of 75 to be $7 \%$ in carriers of the BRCA2 mutation in contrast with $0.85 \%$ for the general population. ${ }^{23}$

Lal et al investigated the frequency of germline $B R C A 1$ and $B R C A 2$ mutations in a prospective study of 102 patients with histologically confirmed pancreatic adenocarcinoma. Although the series was unselected for family history, their cases were selected from a group with a higher proportion of Jewish individuals than the average population $(14 \% v<2 \%) .{ }^{24}$ Mutation analysis was performed in 38 high risk patients; in total there were five germline mutations (13\%) (three BRCA2, one BRCA1, one $p 16$ ).

BRCA2 germline mutation carriers have a 10 -fold higher risk of developing pancreatic cancer than the general population. ${ }^{17}$ The penetrance of the mutation seems to be low and there are likely environmental factors involved, resulting in a rather late onset of the disease. In addition, the exact incidence of $B R C A 2$ germline mutations in the general population has not yet been determined and any screening programme needs this critical information in order to be cost effective. However, there are certain ethnic groups in which particular BRCA2 germline mutations appear to occur often (6174delT in Ashkenazi Jews) and these groups could be an invaluable source for pilot studies to assess the efficacy and cost effectiveness of screening programmes. Families that exhibit clustering of pancreatic and breast cancer may warrant screening for $B R C A 2$ mutations. $^{7}$

Mutation analysis of different grades of precursor lesions (pancreatic intraepithelial neoplasia (PanIN)) for allelic imbalance in germline $B R C A 2$ mutation carriers with pancreatic cancer revealed loss of heterozygosity in one grade 3 PanIN and in none of 13 grade 1 PanIN. These findings indicate that $\mathrm{LOH}$ at the $B R C A 2$ gene locus is not likely to be an early event in tumorigenesis and so the BRCA2 gene does not play a "gatekeeper role" unlike several other genes in inherited cancer syndromes (for instance $A P C$ gene in familial adenomatous polyposis). ${ }^{25}$

\section{Familial atypical multiple mole melanoma syndrome (FAMMM) and $C D K N 2 A / p 16$ germline mutations}

The FAMMM syndrome is an autosomal dominantly inherited syndrome with incomplete penetrance characterised by multiple atypical (dysplastic) nevi, familial clustering of cutaneous malignant melanoma, and increased incidence of extracutaneous cancers. So far, two genes have been implicated in the pathogenesis of malignant melanoma. The first is $C D K N 2 A / p 16$ located at chromosome 9 p21 which encodes a protein (p16) that binds cdk4 and cdk6 (cyclin dependent kinase) and inhibits their abil- ity to form complexes with cyclin D. This in turn prevents the phosphorylation of the retinoblastoma protein and induces growth arrest at the G1 stage of the cell cycle. In contrast with inactivation of the tumour suppressor action of $p 16$, the second known mutation in the pathogenesis of malignant melanoma involves $C D K 4$, a proto-oncogene located at chromosome $12 \mathrm{q} 13$. This mutation has been noted so far in three melanoma prone families. Both the p16 and CDK4 mutations disrupt the same pathway leading to a dysregulated G1/S checkpoint that is commonly affected in cancer.

Early reports identified increased susceptibility to systemic cancers, in addition to melanoma, in a small number of families with FAMMM. ${ }^{26}$ A study of 200 individuals from nine Dutch families with FAMMM revealed an increased frequency of systemic cancers, particularly of the gastrointestinal tract, in three of these families. ${ }^{27}$ Nine of the 43 recorded cancers were pancreatic yielding a ratio of observed to expected frequencies of 13.4. Clearly, FAMMM kindred have a marked increase in pancreatic cancer but not all FAMMM families are prone to pancreatic cancer development. In a study examining the genotype/phenotype relationship in melanoma prone families with $p 16$ and $C D K 4$ germline mutations, similar $p 16$ mutations were noted in families with and without pancreatic cancer, indicating that other factors may be involved in the development of pancreatic cancer. ${ }^{28}$

Goldstein et al found a 22-fold increased risk of pancreatic cancer in multiple melanoma families that harbour germline mutations that impair $p 16$ function, ${ }^{29}$ indicating that this subset of FAMMM families have a higher than expected risk of pancreatic cancer ( 7 observed $v 0.32$ expected). Similar associations between $p 16$ mutation and pancreatic cancer in FAMMM families have been noted by other investigators. ${ }^{13} 30$

In a Swedish study the $p 16$ germline mutation rate was estimated at $19 \%$ in 52 malignant melanoma families (families with at least two cases of malignant melanoma in first or second degree relatives). A common mutation (113insArg) was isolated, indicating a founder mutation for the Swedish population. In this study, families with a germline $p 16$ mutation also had a higher frequency of pancreatic cancer ( 6 cases observed $v 0.16$ expected; $\mathrm{p}<0.0001)$ and breast cancer (8 cases observed $v 2.1$ expected; $p=0.0014) .{ }^{31}$ Thus pancreatic and/or breast cancer families with multiple melanomas should be regarded as candidates for $p 16$ mutation screening.

Melanoma prone families exhibit an earlier than expected age of onset (by two decades compared with the general population) for malignant melanoma while the pancreatic cancers in these families appear at the same late age as in the sporadic form of the disease (typically the seventh decade of life). ${ }^{28}$ Clearly, more melanoma and pancreatic cancer prone families need to be studied. Clinicians should be alert to patients with malignant melanoma and a family history of pancreatic cancer (or vice versa) and a detailed family history should always be obtained. If suspicion arises, referral should be made for genetic testing.

\section{Hereditary pancreatitis and pancreatic cancer}

Hereditary pancreatitis is an autosomal dominant disorder affecting males and females equally. It is characterised by recurrent attacks of abdominal pain and acute pancreatitis that start in childhood (5-10 years old) and leads to the development of chronic pancreatitis by the teenage years. The discovery of a mutation in the cationic trypsinogen gene PRSS1 linked with hereditary pancreatitis shed light on the molecular pathology of this disease. ${ }^{32}$ This mutation appears to make the protein resistant to trypsin cleavage. The result is a protein that is autoactivated with 
inappropriate intracellular accumulation of the proteolytic enzyme trypsin and subsequent digestion of the pancreatic tissue itself. The PRSS1 gene provides an example of how tumours can arise not only from activation of oncogenes and inactivation of tumour suppressor genes but also from mutation of genes that alter the microenvironment of the cell. The process of inflammation and repair is thought to provide a mitogenic stimulus to pancreatic cells. Intriguingly, although it is in the acinar cells that cationic trypsinogen is produced, the cancer that develops subsequently has a ductal phenotype. Much remains to be learnt about the biology of stem cells within the pancreas and about the potential plasticity of differentiation of precursor lesions.

The cumulative lifetime risk of pancreatic cancer in a patient with hereditary pancreatitis has been estimated to be $40 \%$ by the age of 70 , with smokers having a substantially higher risk than non-smokers. ${ }^{33}$ Lowenfels et al have evaluated patients with hereditary pancreatitis and found that non-smokers have about a 40 -fold greater risk of pancreatic cancer than the background population whereas smokers have about a 150 -fold greater risk and the cancer occurs about two decades earlier (personal communication, A Lowenfels). Moreover, the same investigators showed that there was a higher cumulative risk $(75 \%)$ of developing pancreatic cancer if there was paternal transmission of the PRSS1 mutation. Interestingly, the risk of developing pancreatic cancer is substantially higher than the estimated cumulative risk in chronic non-hereditary pancreatitis ( $4 \%$ at 20 years from the diagnosis of chronic pancreatitis). ${ }^{34}$ Unfortunately, failure to detect mutations in the PRSS1 gene in clinically suspect kindred does not exclude its diagnosis. About $30 \%$ of hereditary pancreatitis families test negative for the known mutations. ${ }^{35}$

\section{Hereditary non-polyposis colorectal carcinoma (HNPCC): Lynch syndrome II and pancreatic cancer}

This is an autosomal dominantly transmitted syndrome in which the affected families have an increased risk of developing colorectal adenocarcinoma in association with endometrial, ovarian, and breast cancers, transitional carcinoma of the ureter and renal pelvis, and pancreatic carcinoma. The syndrome is caused by mutations in one of the DNA mismatch repair genes ( $h M S H 2, h M L H 1$, $h P M S 1, h P M S 2)$. These genes encode proteins that correct small DNA errors arising during replication. When inactivated by mutations there is accumulation of replication errors in the DNA that leads to a phenotype called MSI+ or "microsatellite instability". In pancreatic cancer, MSI+ has been shown to occur in about $3.7 \%$ of the tumours studied. $^{36}$ These tumours were characterised by pushing borders, poor differentiation, a syncytial growth pattern, and a wild-type $K R A S$ gene. It seems that this subtype of pancreatic cancer is associated with a better prognosis despite having a poorly differentiated histological phenotype. Certainly, observation of this characteristic pathology combined with wild-type $K R A S$ should raise suspicion of an MSI+ phenotype and the possibility of colonic carcinomas in the affected patient and family. The proportion of familial pancreatic cancers associated with these germline mutations seems to be less than that associated with germline mutations of the $p 16$ and $B R C A 2$ gene but the exact risk of pancreatic cancer in HNPCC kindred remains unknown. ${ }^{17}$

\section{LKB1/STK11 and Peutz-Jeghers syndrome}

Peutz-Jeghers syndrome (PJS) is an autosomal dominant condition characterised by multiple hamartomatous polyps of the gastrointestinal tract and the presence of pigmented lesions (melanin deposits) in the lips, oral mucosa, and digits of the affected individuals. The genetic abnormality responsible for the syndrome is a mutation in the LKB1/ STK11 gene which maps to $19 \mathrm{p} 13$ and encodes a serine/ threonine kinase with an as yet undefined role. ${ }^{37-39}$ The syndrome has been associated with an increased risk of gastrointestinal, gynaecological, breast, and pancreatic cancers. ${ }^{40}$ Approximately $50 \%$ of PJS patients develop some form of cancer. ${ }^{40-43}$ The LKB1/STK1 gene acts as a tumour suppressor gene and is inactivated in $4 \%$ of sporadic pancreatic cancers and $6 \%$ of sporadic biliary adenocarcinomas. ${ }^{43}$ From 53 PJS patients reported in four independent studies, $11 \%$ developed pancreatic adenocarcinoma. ${ }^{43}$ The exact risk that PJS patients carry for pancreatic cancer development is unknown. ${ }^{17}$

\section{Site specific pancreatic cancer families}

At risk members of pancreatic cancer families are either carriers of a known mutation in the germline that produces the same effect as the mutations seen in sporadic cancer or they are carriers of an unknown mutation that predisposes to pancreatic cancer. An interesting family belonging to the latter category has been reported by Evans and colleagues. ${ }^{10}$ This family is characterised by an autosomal dominantly transmitted propensity for pancreatic ductal adenocarcinoma. Members of this family develop diabetes and exocrine pancreatic insufficiency prior to the onset of cancer. Interestingly, members of the family develop pancreatic cancer at an earlier age in each generation (a phenomenon called anticipation). No known germline mutation has been identified so far and some consider this family to represent some form of hereditary pancreatitis. It is possible that in some of the pancreatic cancer families the development of pancreatic cancer may reflect exposure to a common, so far unidentified, carcinogenic factor. Unfortunately, there is no standardised definition for familial pancreatic cancer and experience with pancreatic cancer surveillance in high risk groups is still limited. The essence of screening for cancer of any kind involves application of diagnostic modalities in asymptomatic individuals when the disease ideally is still at the early stage of dysplasia or in situ carcinoma. The diagnostic modalities should offer high sensitivity and specificity for detecting the dysplastic lesions, be non-invasive (if possible), and at a low cost. At this stage of the disease the benefit for the patient is maximal providing there are management strategies available with an acceptable morbidity and mortality.

\section{Who is at risk?}

Identification of the target population is essential in designing screening programmes. The family members of known cancer syndromes such as the familial breast cancer syndrome associated with $B R C A 2$ mutation, FAMMM syndrome, PJS, HNPCC syndrome, and patients with hereditary pancreatitis certainly have a higher risk of pancreatic cancer than the average population and logically are candidates for screening. Also, the University of Washington criteria identify individuals with a high risk who do not belong to any of the so far identified family cancer syndromes but should be considered candidates for surveillance. Certain ethnic groups such as Ashkenazi Jews may also be considered as candidates for screening. Patients with hereditary pancreatitis are a subgroup of high risk patients who may benefit from screening. These patients offer a special technical challenge because the cancers arise in the setting of chronic pancreatitis; using standard imaging modalities it is difficult to distinguish early cancers from chronic inflammatory changes. 


\section{When to start screening?}

Although there are no definite data, experts with experience in familial pancreatic cancer surveillance variously suggest commencement of screening at least a decade before the earliest age of onset of pancreatic cancer in an affected family, at the age of 50 years, or when symptoms develop. Individuals should remain under surveillance for as long as they continue to remain surgical candidates in case pancreatic cancer is discovered ${ }^{17}$ In the case of hereditary pancreatitis, it is suggested that screening commence as early as 30 years old. ${ }^{44}$

\section{How to screen?}

Our progress in defining the molecular changes associated with pancreatic cancer during the last decade and technical improvements in imaging modalities have not led to an appreciable improvement in the early diagnosis of pancreatic cancer. The vast majority of cases are still discovered at a late stage, either being locally advanced or having already metastasised. However, we now have a fair idea of the molecular events that accumulate in cells and lead to the acquisition of a malignant phenotype.

Mutational activation of the $K R A S$ is an early event in pancreatic carcinogenesis and is found in up to $90 \%$ of pancreatic adenocarcinomas. ${ }^{45}$ This fact combined with the relative simplicity of the techniques needed to identify mutations makes KRAS a possible molecular marker. KRAS mutations have been detected in plasma, stools, duodenal aspirate, pancreatic juice, and ERCP brush samples with a sensitivity of 33-67\% and specificity approaching $100 \%{ }^{46-51}$ However, the occurrence of KRAS mutations in some patients with hyperplastic lesions (PanIN I) associated with chronic pancreatitis limits the usefulness of $K R A S$ mutation as a screening tool. ${ }^{52}$

Lately, attention has been focused on the increased telomerase activity measured in pancreatic juice from pancreatic cancer cases. The high sensitivity $(80 \%)$ and $100 \%$ specificity makes this technique a potential screening tool, particularly as there are reports of increased telomerase activity in the pancreatic juice of patients well before the detection of pancreatic cancer. ${ }^{53}{ }^{54}$ These findings need to be verified with further studies before definite conclusions can be drawn.

Recent studies identified loss of heterozygosity ( $\mathrm{LOH}$ ) in preneoplastic intraductal lesions for the tumour suppressor genes $p 16, P 53$, and $M A D H 4$. There was a suggestion of p16 accumulating earlier in preneoplastic intraductal lesions whereas P53 and MADH4 mutations exhibited a tendency to accumulate in lesions with nuclear atypia. ${ }^{55}$ Although LOH of one allele does not necessarily imply mutation of the other allele (resulting in total loss of functional protein) these results are potentially interesting in designing screening strategies but they need to be confirmed in larger scale.

The role of tumour markers in pancreatic cancer has been comprehensively reviewed by Lamerz. ${ }^{56}$ CA $19-9$ still seems the most important conventional molecular marker for pancreatic adenocarcinoma with a sensitivity of $68-92 \%$ and a specificity of $65-92 \%$. Its usefulness so far has been limited to assessing response to treatment and detection of relapse.

\section{How often to screen?}

There are no clear data available on the optimal interval for pancreatic cancer surveillance in high risk groups but 6-12 month intervals have been suggested for high risk patients who have abnormalities on screening tests such as endoscopic ultrasound (EUS) ${ }^{17}$ Patients who have negative findings at screening may have longer follow up intervals (12-24 months).

\section{Imaging modalities for detection of pancreatic} cancer

Spiral computed tomography is the current state of the art for investigation of pancreatic cancer and it is the test that is almost always obtained during the diagnostic workup of a patient with a suspicious pancreatic lesion, but its resolution is limited for small lesions $(1-2 \mathrm{~cm})$. It has a sensitivity of $89 \%$ and an overall accuracy of $70 \%$ for assessing resectability. ${ }^{57}$ Early experience suggests that this test is ineffective for surveillance. ${ }^{16} 17$

Magnetic resonance imaging and magnetic resonance cholangiopancreatography (MRCP) are relatively new diagnostic modalities used in pancreatic imaging and further data need to be collected before the sensitivity and specificity in pancreatic cancer detection can be accurately assessed. MRCP is expected to supersede the use of diagnostic endoscopic retrograde cholangiopancreatography (ERCP) as it is non-invasive and has no complications. ${ }^{58}$ The sensitivity of MRCP in diagnosing pancreatic cancer has been reported to be as high as $84 \%$, together with $97 \%$ specificity. ${ }^{59}$ However, early reports suggest that so far the use of MRCP has not improved the diagnosis of early pancreatic carcinoma. ${ }^{60}$

EUS has $95 \%$ sensitivity, $80 \%$ specificity, $95 \%$ positive predictive value, and $88 \%$ negative predictive value in detecting malignant lesions in the pancreas. ${ }^{61}$ EUS has been shown to be effective in identifying early lesions in pancreatic cancer surveillance programmes and is considered the first step in investigating asymptomatic high risk individuals. ${ }^{17}$ It is relatively non-invasive but it requires considerable experience. Findings include heterogeneous parenchyma with 1-2 mm scattered echogenic foci, hypoechoic nodules $2-4 \mathrm{~mm}$ in diameter, hyperechoic walls in the main duct, and discrete masses, while some of these abnormalities are similar to those seen in chronic pancreatitis. If a mass is present, EUS can be combined with fine needle aspiration for a guided biopsy to confirm the diagnosis but a negative result does not exclude cancer. ${ }^{61}$

ERCP has a sensitivity of $90-97 \%$ and specificity of $81-90 \%$ in pancreatic cancer. ${ }^{62}$ Its usefulness in screening programmes has been shown in detecting changes in the ductal system similar to those seen in chronic pancreatitis. ${ }^{16}$ These changes have subsequently been shown to correlate with dysplasia in the pancreas (PanIN grade 2 and 3 ). However, ERCP has some drawbacks: the changes would not be interpretable in patients with hereditary pancreatitis, and it remains an invasive test with a potential morbidity ( $4 \%$ major complications) or even mortality $(0.2 \%$ for diagnostic ERCP). ${ }^{63}$

Positron emission tomography with fluorodeoxyglucose is based on an increased metabolic rate for glucose observed in cancer cells. Experience is still limited but sensitivity up to $85 \%$ and specificity up to $84 \%$ have been reported in pancreatic cancer detection. ${ }^{6465}$ Its role in detecting small tumours is not yet established, and its very high cost makes it an unlikely tool to be used as a pancreatic cancer surveillance test.

\section{Conclusions}

Clinicians should be aware of the disorders associated with increased susceptibility to pancreatic cancer and initiate appropriate investigations when the symptoms point towards a pancreatic origin. More importantly these disorders require the development of screening strategies to attempt to detect early cases with the highest chances of cure. So far there is no consensus on when and how to screen these groups for pancreatic cancer. However, there is a need to develop such strategies for the high risk pancreatic cancer groups, exploiting the advances of the last decade in molecular pathology and imaging to their full 
capacity. Screening programmes for pancreatic cancer will most likely be costly, as the diagnostic techniques are sophisticated and exceed the capabilities of a typical general hospital. Hepatopancreaticobiliary units with an interest in pancreatic surgery and pancreatic cancer seems to be the most appropriate environment for these programmes which should be carried by a multidisciplinary team including pancreatic surgeons, gastroenterologists, and molecular pathologists. Genetic counselling should be offered to every patient prior to enrolment in screening programmes.

E EFTHIMIOU T CRNOGORAC-JURCEVIC N R LEMOINE

ICRF Molecular Oncology Unit,

Imperial College School of Medicine,

Hammersmith Hospital, London, UK

T A BRENTNALL

Division of Gastroenterology,

University of Washington, Seattle 98195, USA

Correspondence to: Professor N R Lemoine, ICRF Molecular Oncology Unit, Department of Cancer Medicine, Imperial College School of Medicine,

Hammersmith Campus, London W12 0NN, UK. n.lemoine@icrf.icnet.uk

1 Schnall SF, Macdonald JS. Chemotherapy of adenocarcinoma of the pancreas. Semin Oncol 1996;23:220-8.

2 Silverman DT, Dunn JA, Hoover RN, et al. Cigarette smoking and pancreas cancer: a case-control study based on direct interviews. I Natl Cancer Inst 1994;86:1510-16.

3 Silverman DT, Schiffman M, Everhart J, et al. Diabetes mellitus, other medical conditions and familial history of cancer as risk factors for pancreatic cancer. Br F Cancer 1999;80:1830-7.

4 Lemoine NR. Molecular advances in pancreatic cancer. Digestion 1997;58: 550-6.

5 Ghadirian P, Boyle P, Simard A, et al. Reported family aggregation of pancreatic cancer within a population-based case-control study in the Francophone community in Montreal, Canada. Int $\mathcal{F}$ Pancreatol 1991;10:183-96.

6 Fernandez E, La Vecchia C, D'Avanzo B, et al. Family history and the risk of liver, gallbladder, and pancreatic cancer. Cancer Epidemiol Biomarkers Prev 1994;3:209-12.

7 Hruban RH, Petersen GM, Goggins M, et al. Familial pancreatic cancer.

Ann Oncol, 1999;10(suppl 4):69-73.
8 Danes BS, Lynch HT. A familial aggregation of pancreatic cancer. An in Danes BS, Lynch HT. A familial aggreg
vitro study. I $A M A$ 1982;247:2798-802.

9 Ehrenthal D, Haeger L, Griffin T, et al. Familial pancreatic adenocarcinoma in three generations. A case report and a review of the literature. Cancer 1987;59:1661-4.

10 Evans JP, Burke W, Chen R, et al. Familial pancreatic adenocarcinoma: association with diabetes and early molecular diagnosis. F Med Genet 1995; 32:330-5.

11 Lynch HT, Fitzsimmons ML, Smyrk TC, et al. Familial pancreatic cancer: clinicopathologic study of 18 nuclear families. Am $\mathcal{F}$ Gastroenterol 1990;85: 54-60.

12 Lynch HT, Fusaro L, Lynch JF. Familial pancreatic cancer: a family study. Pancreas 1992;7:511-15.

13 Whelan AJ, Bartsch D, Goodfellow PJ. Brief report: a familial syndrome of pancreatic cancer and melanoma with a mutation in the CDKN2 tumor-suppressor gene. N Engl f Med 1995;333:975-7.

14 Levin B. An overview of preventive strategies for pancreatic cancer. Ann Oncol 1999;10(suppl 4):193-6.

15 Lynch HT, Smyrk T, Kern SE, et al. Familial pancreatic cancer: a review. Semin Oncol 1996;23:251-75.

16 Brentnall TA, Bronner MP, Byrd DR, et al. Early diagnosis and treatment of pancreatic dysplasia in patients with a family history of pancreatic cancer. Ann Intern Med 1999;131:247-5

17 Brentnall TA. Cancer surveillance of patients from familial pancreatic cancer kindreds. Med Clin North Am 2000;84:707-18.

18 Roa BB, Boyd AA, Volcik K, et al. Ashkenazi Jewish population frequencies for common mutations in BRCA1 and BRCA2. Nat Genet 1996;14:185-7.

19 Tonin P, Weber B, Offit K, et al. Frequency of recurrent BRCA1 and BRCA2 mutations in Ashkenazi Jewish breast cancer families. Nat Med 1996;2:1179-83.

20 Thorlacius S, Olafsdottir G, Tryggvadottir L, et al. A single BRCA2 mutation in male and female breast cancer families from Iceland with varied cancer phenotypes. Nat Genet 1996;13:117-19.

21 Phelan CM, Lancaster JM, Tonin P, et al. Mutation analysis of the BRCA2 gene in 49 site-specific breast cancer families. Nat Genet 1996;13:120-2.

22 Goggins M, Schutte M, Lu J, et al. Germline BRCA2 gene mutations in patients with apparently sporadic pancreatic carcinomas. Cancer Res 1996; 56:5360-4.

23 Ozcelik H, Schmocker B, Di Nicola N, et al. Germline BRCA2 6174delT mutations in Ashkenazi Jewish pancreatic cancer patients. Nat Genet 1997; 16:17-18

24 Lal G, Liu G, Schmocker B, et al. Inherited predisposition to pancreatic adenocarcinoma: role of family history and germ-line p16, BRCA1, and BRCA2 mutations. Cancer Res 2000;60:409-16.

25 Goggins M, Hruban RH, Kern SE. BRCA2 is inactivated late in the development of pancreatic intraepithelial neoplasia: evidence and implications. Am F Pathol 2000;156:1767-71.

26 Lynch HT, Fusaro RM, Kimberling WJ, et al. Familial atypical multiple mole-melanoma (FAMMM) syndrome: segregation analysis. 7 Med Genet 1983;20:342-4.

27 Bergman W, Watson P, de Jong J, et al. Systemic cancer and the FAMMM syndrome. Br f Cancer 1990;61:932-6.
28 Goldstein AM, Struewing JP, Chidambaram A, et al. Genotype-phenotype relationships in US melanoma-prone families with CDKN2A and CDK4 mutations. I Natl Cancer Inst 2000;92:1006-10.

29 Goldstein AM, Fraser MC, Struewing JP, et al. Increased risk of pancreatic cancer in melanoma-prone kindreds with p16INK4 mutations. N Engl f Med 1995;333:970-4

30 Lal G, Liu L, Hogg D, et al. Patients with both pancreatic adenocarcinoma and melanoma may harbor germline CDKN2A mutations. Genes Chromosomes Cancer 2000;27:358-61.

31 Borg A, Sandberg $\mathrm{T}$, Nilsson $\mathrm{K}$, et al. High frequency of multiple melanomas and breast and pancreas carcinomas in CDKN2A mutationpositive melanoma families. $\mathcal{F}$ Natl Cancer Inst 2000;92:1260-6.

32 Whitcomb DC, Gorry MC, Preston RA, et al. Hereditary pancreatitis is caused by a mutation in the cationic trypsinogen gene. Nat Genet 1996;14: $141-5$.

33 Lowenfels AB, Maisonneuve P, DiMagno EP, et al. Hereditary pancreatitis and the risk of pancreatic cancer. International Hereditary Pancreatitis Study Group. $\mathcal{F}$ Natl Cancer Inst 1997;89:442-6.

34 Lowenfels AB, Maisonneuve P, Cavallini G, et al. Pancreatitis and the risk of pancreatic cancer. International Pancreatitis Study Group. $N$ Engl f Med 1993;328:1433-7.

35 Steinberg WM, Barkin J, Bradley EL III, et al. Workup of a patient with familial pancreatic cancer. Pancreas 1999;18:219-24.

36 Goggins M, Offerhaus GJ, Hilgers W, et al. Pancreatic adenocarcinomas with DNA replication errors (RER+) are associated with wild-type K-ras and characteristic histopathology. Poor differentiation, a syncytial growth pattern, and pushing borders suggest RER+. Am f Pathol 1998;152:1501-7.

37 Hemminki A, Tomlinson I, Markie D, et al. Localization of a susceptibility locus for Peutz-Jeghers syndrome to $19 \mathrm{p}$ using comparative genomic hybridization and targeted linkage analysis. Nat Genet 1997;15:87-90.

38 Hemminki A, Markie D, Tomlinson I, et al. A serine/threonine kinase gene defective in Peutz-Jeghers syndrome. Nature 1998;391:184-7.

39 Jenne DE, Reimann H, Nezu J, et al. Peutz-Jeghers syndrome is caused by mutations in a novel serine threonine kinase. Nat Genet 1998;18:38-43.

40 Boardman LA, Thibodeau SN, Schaid DJ, et al. Increased risk for cancer in patients with the Peutz-Jeghers syndrome. Ann Intern Med 1998;128:896-9.

41 Hizawa K, Iida M, Matsumoto T, et al. Cancer in Peutz-Jeghers syndrome. Cancer 1993;72:2777-81.

42 Giardiello FM, Welsh SB, Hamilton SR, et al. Increased risk of cancer in the Peutz-Jeghers syndrome. N Engl f Med 1987;316:1511-14.

$43 \mathrm{Su}$ GH, Hruban RH, Bansal RK, et al. Germline and somatic mutations of the STK1 1/LKB1 Peutz-Jeghers gene in pancreatic and biliary cancers. Am f Pathol 1999;154:1835-40.

44 Finch MD, Howes N, Ellis I, et al. Hereditary pancreatitis and familial pancreatic cancer. Digestion 1997;58:564-9.

45 Hall PA, Lemoine NR. Models of pancreatic cancer. Cancer Surv 1993;16: $135-55$.

46 Watanabe $\mathrm{H}, \mathrm{Ha} \mathrm{A}, \mathrm{Hu} \mathrm{YX}$, et al. K-ras mutations in duodenal aspirate without secretin stimulation for screening of pancreatic and biliary tract carcinoma. Cancer 1999;86:1441-8.

47 Kondo H, Sugano K, Fukayama N, et al. Detection of point mutations in the K-ras oncogene at codon 12 in pure pancreatic juice for diagnosis of pancreatic carcinoma. Cancer 1994;73:1589-94.

48 Kondo H, Sugano K, Fukayama N, et al. Detection of K-ras gene mutations at codon 12 in the pancreatic juice of patients with intraductal papillary mucinous tumors of the pancreas. Cancer 1997;79:900-5.

49 Iguchi $\mathrm{H}$, Sugano K, Fukayama N, et al. Analysis of Ki-ras codon 12 mutations in the duodenal juice of patients with pancreatic cancer. Gastroenterology 1996;110:221-6.

50 Tada M, Omata M, Kawai S, et al. Detection of ras gene mutations in pancreatic juice and peripheral blood of patients with pancreatic adenocarcinoma. Cancer Res 1993;53:2472-4.

51 Watanabe $\mathrm{H}$, Sawabu N, Ohta $\mathrm{H}$, et al. Identification of K-ras oncogene mutations in the pure pancreatic juice of patients with ductal pancreatic cancers. Fpn f Cancer Res 1993;84:961-5.

52 Rivera JA, Rall CJ, Graeme-Cook F, et al. Analysis of K-ras oncogene mutations in chronic pancreatitis with ductal hyperplasia. Surgery 1997;121:42-9.

53 Suehara N, Mizumoto K, Muta T, et al. Telomerase elevation in pancreatic ductal carcinoma compared to nonmalignant pathological states. Clin Cancer Res 1997;3:993-8.

54 Uehara H, Nakaizumi A, Tatsuta M, et al. Diagnosis of pancreatic cancer by detecting telomerase activity in pancreatic juice: comparison with $\mathrm{K}$-ras mutations. Am ₹ Gastroenterol 1999;94:2513-18.

55 Heinmoller E, Dietmaier W, Zirngibl H, et al. Molecular analysis of microdissected tumors and preneoplastic intraductal lesions in pancreatic carcinoma. Am 7 Pathol 2000;157:83-92.

56 Lamerz R. Role of tumour markers, cytogenetics. Ann Oncol 1999;10(supp1 4):145-9.

57 Bluemke DA, Cameron JL, Hruban RH, et al. Potentially resectable pancreatic adenocarcinoma: spiral CT assessment with surgical and pathologic correlation. Radiology 1995;197:381-5.

58 Ferrucci JT. MRI and MRCP in pancreaticobiliary malignancy. Ann Oncol 1999;10(suppl 4):18-19.

59 Adamek HE, Albert J, Breer H, et al. Pancreatic cancer detection with magnetic resonance cholangiopancreatography and endoscopic retrograde cholangiopancreatography: a prospective controlled study. Lancet 2000; 356:190-3.

60 Furukawa H, Muramatsu Y, Moriyama N. Does MR cholangiopancreatography contribute to the early diagnosis of pancreatic carcinoma? Pancreas 1999;19:408-9.

61 Baron PL, Aabakken LE, Cole DJ, et al. Differentiation of benign from malignant pancreatic masses by endoscopic ultrasound. Ann Surg Oncol 1997;4:639-43.

62 Martin SP, Ulrich CD II. Pancreatic cancer surveillance in a high-risk cohort. Is it worth the cost? Med Clin North Am 2000;84:739-47.

63 Loperfido S, Angelini G, Benedetti G, et al. Major early complications from diagnostic and therapeutic ERCP: a prospective multicenter study. Gastrointest Endosc 1998;48:1-10.

64 Zimny M, Buell U. 18FDG-positron emission tomography in pancreatic cancer. Ann Oncol 1999;10(suppl 4):28-32.

65 Keogan MT, Tyler D, Clark L, et al. Diagnosis of pancreatic carcinoma: role of FDG PET. AfR Am $\mathcal{f}$ Roentgenol 1998;171:1565-70. 